SHORT REPORT

\title{
A novel multimedia tool to improve bedside teaching of cardiac auscultation
}

\author{
A Woywodt, A Herrmann, J T Kielstein, H Haller, M Haubitz, H Purnhagen
}

Postgrad Med J 2004;80:355-357. doi: 10.1136/pgmj.2003.014944

Training in cardiac auscultation is a core element of undergraduate teaching but recent studies have documented a remarkable decline in auscultatory skills. Therefore there is an interest in new ways to teach cardiac auscultation. In analogy to phonocardiography, an electronic system for simultaneous auscultation and visualisation of murmurs was sought. For this purpose, an electronic stethoscope was linked to a laptop computer and software created to visualise auscultatory findings. In a preliminary trial in undergraduate students, this approach greatly facilitated teaching. Amalgamating traditional phonocardiography with a multimedia approach, this system represents a novel tool for bedside teaching of cardiac auscultation.

$\mathrm{R}$ ecent surveys have demonstrated poor proficiency in cardiac auscultation ${ }^{1}$ with surprisingly few differences between countries. A new generation of electronic stethoscopes has recently become available, albeit with questionable benefits for the experienced auscultator. ${ }^{2}$ The use of these devices for teaching, however, has not been appreciated. In particular, newer versions also host a saving function and permit infrared transmission of sound files. Being involved in undergraduate teaching, we were interested to link an electronic stethoscope to a laptop computer and thus assemble a unique teaching tool that permits visualisation and amplification of auscultatory findings.

\section{METHODS}

We used a Littmann 4000 stethoscope (Littmann, Oakdale, Minnesota, USA), and a Compaq Armada lo0S (Compaq, Houston, Texas, USA) with infrared transponder. The Littmann 4000 software stores audio files in $\mathrm{a}^{*} . \mathrm{e} 4 \mathrm{k}$ format, a 16 bit signed integer (little endian) code not recognised by common audio software. A 51 byte header precedes each file. With regard to easy access, we converted these files to *.wav. Free conversion software is available from various sites (such as www.tsp.ece.mcgill.ca/Docs/Software/index.html accessed 13 February 2003). Converted *.wav files were visualised with Goldwave, an audio editor that is also freely available (www.goldwave.com, accessed 13 February 2002). Various filter settings were assessed for conversion to ${ }^{*}$.wav files. Selecting an $8000 \mathrm{~Hz}$ sampling rate usually resulted in excellent sound quality. CopyAudio, however, is a DOS command line application for which a wrapper utility for Windows was constructed. The program can be used in two ways, namely to convert *e4k files "manually" or to convert all audio files that come in via the infrared port. Converted *.wav files were viewed after magnification. A $1500 \mathrm{~Hz}$ lowpass filter was used for noise reduction. The system was first used in a group of 12 medical students who participated in a mandatory six month course of history taking and physical examination. We examined three patients with aortic stenosis, one with aortic regurgitation, one with pericarditis of unknown origin, two with mitral regurgitation, one with mitral stenosis, and one young patient with a loud third heart sound but without evidence of structural heart disease.

\section{RESULTS}

In our hands, the "electronic phonocardiogram" described here performed extremely well as a teaching tool. In essence, the opportunity to auscultate while watching the electronic phonocardiogram at the same time proved most valuable (fig 1). We started with normal heart sounds and demonstrated the splitting of S2, which we regarded as difficult to grasp for beginners. With electronic amplification and visualisation, the students were able to understand this phenomenon. Our system lacks simultaneous recording of the electrocardiogram (ECG). In general, however, S1 and S2 could be identified quite easily and pathological sounds such as a third heart sound could thus be differentiated (fig 2A). The lack of concomitant ECG therefore did not prove a significant drawback. A third heart sound is also difficult to detect for beginners. Yet students found it easy to detect after they had appreciated its temporal relation to S1 and S2. By contrast, aortic stenosis is relatively easy to detect, although the time to peak and the loudness of the second heart sound is not. When displayed with the electronic phonocardiogram, these findings were also appreciated, as was the crescendodecrescendo shape of the murmur (fig 2B). Another "difficult murmur", often missed entirely by beginners, is aortic regurgitation (fig $2 \mathrm{C}$ ). So too is mitral stenosis, while mitral regurgitation is relatively easy to pick up. Both the low frequency diastolic murmur of mitral stenosis and the opening snap were nicely displayed (fig 2D). The system also permitted storing of murmurs for later comparison. By virtue of the *.wav format, the sound files could be played on almost every computer with Windows Media Player or any other audio program.

\section{DISCUSSION}

The decline of clinical skills has been emphasised more often than not $^{3}$ and many suggestions have been made to modernise cardiac auscultation and employ multimedia systems. ${ }^{4}$ While collections of murmurs and sounds or simulators are certainly valuable, ${ }^{5}$ the importance of bedside teaching should not be underestimated. Our approach is an extension of a system described by Tavel et al, ${ }^{6}$ but adds the opportunity to view and amplify sounds and murmurs on the screen during auscultation at the bedside. Both studies continue the remarkable tradition of phonocardiography ${ }^{78}$ with a common philosophy of understanding murmurs by visualisation. Our system has several advantages over conventional phonocardiography. First, sounds and murmurs can be amplified or played at lower speed to permit optimal visualisation and thereby overcome limitations of the human auditory system. In particular, inexperienced auscultators 


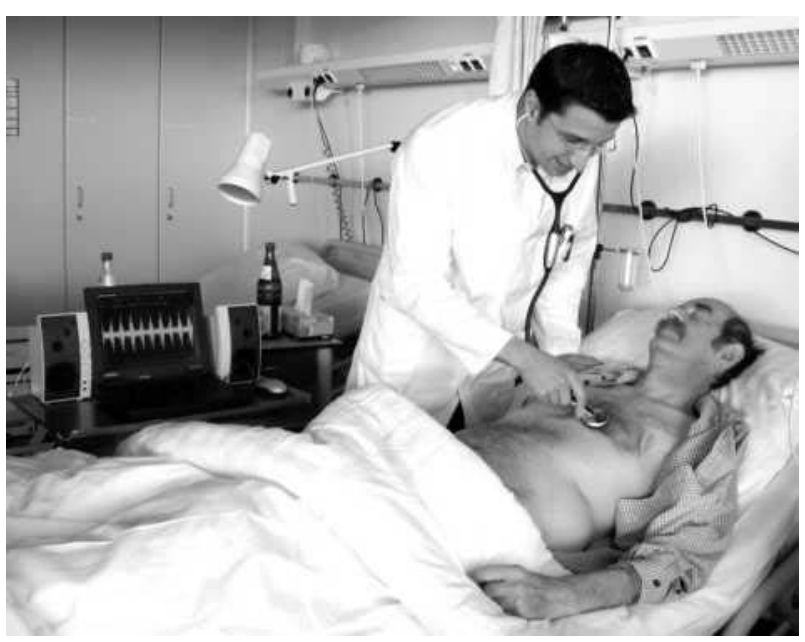

Figure 1 Typical setup with one student auscultating the patient. Other students can view the phonocardiogram as displayed on the computer and listen to the murmur simultaneously.

tend to miss low frequency sounds. ${ }^{9}$ Also, the temporal relationship of murmurs and sounds is easily appreciated. Finally, sound files can be stored, viewed later, or transferred via email. ${ }^{10}$ Archiving of cases for teaching purposes is another possibility. Availability and cost are also important issues. Phonocardiography is no longer available in our institution and in many other teaching hospitals. In contrast,

\section{Summary points}

- Cardiac auscultation is an essential part of clinical examination; recent studies, however, have demonstrated a remarkable decline in auscultatory skills.

- Teaching cardiac auscultation has been augmented with multimedia approaches before, albeit these studies have focused on learning from collections of sounds and murmurs.

- Electronic stethoscopes are becoming increasingly popular; their use for teaching cardiac auscultation has so far not been appreciated.

- We have linked an electronic stethoscope with a laptop computer to permit bedside "electronic phonocardiography" in order to facilitate the teaching of cardiac auscultation.

electronic stethoscopes are becoming increasingly popular and large numbers of laptop computers are also in use. In this setting, our system does not account for any extra costs. Notably, the software used here is freeware from internet sources.

In conclusion, we have reported setup and technical features of a simple new system for the bedside teaching of cardiac auscultation as well as our first experience with this approach. This system, we believe, holds considerable promise with regard to undergraduate teaching. We would
A

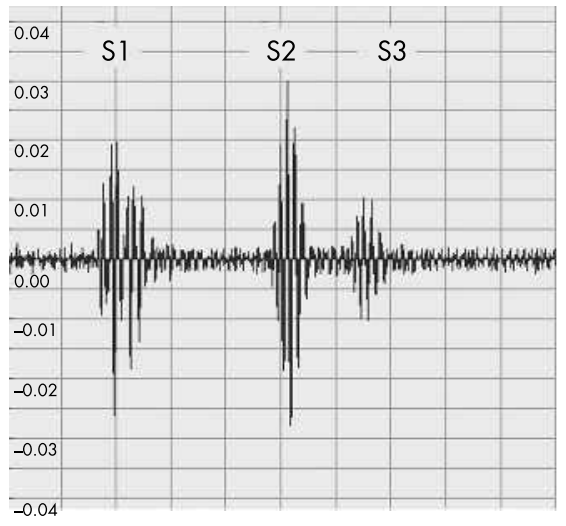

C

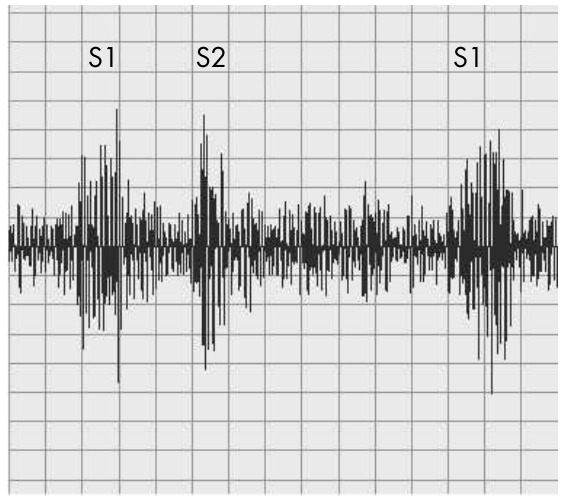

B

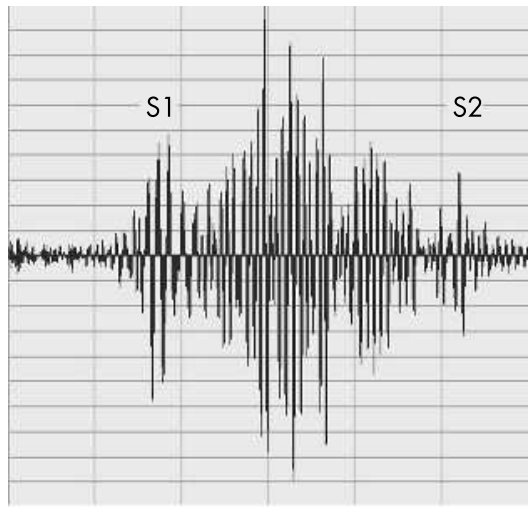

D

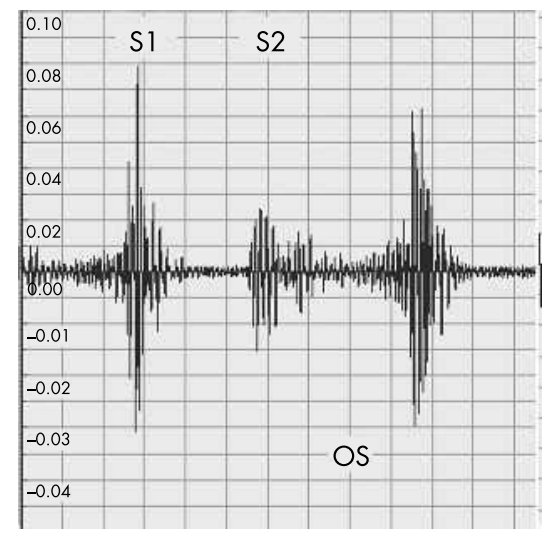

Figure 2 (A) Third heart sound in a young healthy subject. (B) Severe aortic stenosis in an 82 year old woman (aortic valve orifice area $0.8 \mathrm{~cm}^{2}$, peak gradient $83 \mathrm{~mm} \mathrm{Hg}$ ). (C) Severe aortic regurgitation due to aortic root enlargement with concomitant aortic dissection; the band shaped diastolic murmur is easily appreciated. (D) Mitral stenosis; OS denotes the opening snap. $\mathrm{S} 1$ is rather loud as is often observed in mitral stenosis. 
like to encourage others to use our approach and add further improvements.

\section{ACKNOWLEDGEMENTS}

We are indebted to Friedrich C Luft (Franz-Volhard-Clinic, Charité Campus Berlin-Buch, Berlin, Germany) for help with the manuscript. We also appreciated the help of Frank Streiber, MS, and Maik Schroeder, MS. We are also indebted to the students and patients who agreed to participate in this study.

\section{Authors' affiliations}

A Woywodt, A Herrmann, J T Kielstein, H Haller, M Haubitz, Division of Nephrology, Department of Medicine, Hannover Medical School, Hannover, Germany

H Purnhagen, Information Technology Laboratory, University of Hannover, Hannover, Germany

Conflict of interest: There is no conflict of interest, particularly no connection whatsoever with Littmann $3 \mathrm{M}$, the manufacturer of the stethoscope.

Correspondence to: Dr A Woywodt, Department of Medicine, Hannover Medical School, Carl-Neuberg-Strasse 1, 30625 Hannover, Germany; Woywodt.Alexander@MH-Hannover.de
Received 12 September 2003

Accepted 17 October 2003

\section{REFERENCES}

1 Mangione S, Nieman LZ, Gracely E, et al. The teaching and practice of cardiac auscultation during internal medicine and cardiology training. A nationwide survey. Ann Intern Med 1993;119:47-54.

2 Schmedtie JF Jr. Hearing is believing. Lancet 2002;359:1078.

3 Woywodt A, Hofer M, Pilz B, et al. Cardiopulmonary auscultation: duo for strings-Opus 99. Arch Intern Med 1999;159:2477-9.

4 Waugh RA, Mayer JW, Ewy GA, et al. Multimedia computer-assisted instruction in cardiology. Arch Intern Med 1995; 155:197-203.

5 Jones JS, Hunt SJ, Carlson SA, et al. Assessing bedside cardiologic examination skills using "Harvey," a cardiology patient simulator. Acad Emerg Med 1997;4:980-5.

6 Tavel ME, Brown DD, Shander D. Enhanced auscultation with a new graphic display system. Arch Intern Med 1994;154:893-8.

7 Verheugt FW. Frans C Donders. Clin Cardiol 1998;21:371-2.

8 Silverman ME. Aubrey Leatham: twentieth century pioneer in auscultation. Clin Cardiol 1999;22:155-7.

9 Sapira JD. The art and science of bedside diagnosis. Baltimore: Williams and Wilkins, 1990:309.

10 Dahl LB, Hasvold P, Arild E, et al. Heart murmurs recorded by a sensor based electronic stethoscope and e-mailed for remote assessment. Arch Dis Child 2002;87:297-301. 